\title{
Estructura y diversidad de plantas leñosas de la selva mediana subcaducifolia en el centro de Veracruz, México
}

\section{Structure and diversity of woody plants of the semideciduous forest in central Veracruz, Mexico}

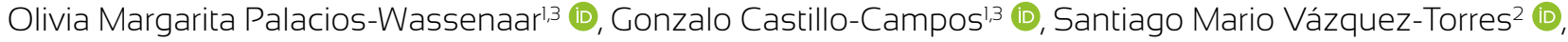 \\ María Elena Medina-Abreo' (D)
}

1 Instituto de Ecología, A.C., Red de Biodiversidad y Sistemática, carretera antigua a Coatepec Núm. 351, El Haya, 91070 Xalapa, Veracruz, México.

2 Centro de Investigaciones Tropicales (CITRO), José María Morelos 44, Zona Centro, 91000 Xalapa, Veracruz, México.

3 Autores para la correspondencia: olivia.palacios@gmail.com; gonzalo. castillo@inecol.mx

Recibido: 12 de agosto de 2017. Revisado: 31 de octubre de 2017.

Aceptado: 12 de enero de 2018.

Primero en línea: 29 de mayo de 2018.

Publicado: 1 de julio de 2018.

Citar como:

Palacios-Wassenaar, O. M., G. CastilloCampos, S. M. Vázquez-Torres y M. E. Medina-Abreo. 2018. Estructura y diversidad de plantas leñosas de la selva mediana subcaducifolia en el centro de Veracruz, México. Acta Botanica Mexicana 124: 85-104. DOI: 10.21829/ abml24.2018.1279

DOI:

10.21829/abml24.2018.1279

\section{Resumen:}

Antecedentes y Objetivos: El estudio de las selvas tropicales es de gran interés debido a su alta biodiversidad, relacionada con la variabilidad del ambiente, que incrementa la diversidad y la especificidad de sus componentes. Este estudio presenta una evaluación de la estructura, riqueza y diversidad de especies de angiospermas leñosas de la selva mediana subcaducifolia ( $\mathrm{SMsC}$ ) en el centro de Veracruz, con el objetivo de contribuir al conocimiento de sus características e identificar áreas de alto valor para la conservación de los escasos fragmentos de vegetación primaria que aún existen en la zona.

Métodos: Se delimitaron 67 parcelas de muestreo de $100 \mathrm{~m}^{2}$, distribuidas en cinco sitios de estudio en los municipios Jalcomulco y Tlaltetela. En cada parcela se registraron y midieron todos los individuos con DAP $\geq 5 \mathrm{~cm}$ y se estimaron sus valores de cobertura, densidad, área basal y altura, así como los valores de diversidad $\alpha$ (riqueza total, riqueza por parcela e índice $\alpha$ de Fisher) y los valores de diversidad $\beta$ (matriz de similitud con coeficiente de Jaccard y porcentaje de similitud), además de la importancia ecológica de las especies mediante el Valor de Importancia Relativa de Curtis (VIR).

Resultados clave: Se registraron 98 especies de plantas leñosas, agrupadas en 83 géneros y 35 familias de angiospermas. Las familias más diversas fueron Fabaceae y Euphorbiaceae. La forma biológica predominante $(75.5 \%)$ fueron los árboles. La selva tropical mostró una alta riqueza y elevada tasa de recambio de especies. Las especies dominantes de acuerdo con el VIR fueron Resinanthus aromaticus y Brosimum alicastrum, aunque el conjunto de las seis especies dominantes fue muy variable entre los sitios de estudio. Conclusiones: La presencia de especies clave como Brosimum alicastrum, Resinanthus aromaticus y Sapranthus microcarpus permitió identificar los elementos característicos de la SMsC y sus ecotonos con la selva mediana subperennifolia y la selva baja caducifolia.

Palabras clave: conservación, valor de importancia relativa, recambio de especies, Resinanthus aromaticus, selvas tropicales estacionalmente secas, vegetación primaria.

\section{ABSTRACT:}

Background and Aims: The study of tropical forests is of great interest due to its high biodiversity related to the variability of the environment, which increases the diversity and specificity of its components. This study presents an evaluation of the structure, richness and diversity of woody angiosperms of the semideciduous forest (SMsC) in the center of the state of Veracruz, with the aim of contributing to their knowledge and to identify areas of high value for the conservation of the few fragments of primary vegetation that still exist in the area. Methods: Sixty-seven plots of $100 \mathrm{~m}^{2}$ each were delineated, distributed in five study sites in the municipalities of Jalcomulco and Tlaltetela. In each plot, all individuals with $\mathrm{DBH} \geq 5 \mathrm{~cm}$ were registered and measured and coverage, density, basal area and height were estimated, as well as the values of $\alpha$ diversity (total richness, richness per plot and $\alpha$ Fisher index), $\beta$ diversity (similarity matrix with Jaccard and \% similarity), and as the species ecological importance by the Curtis Relative Importance Value (RIV).

Key results: Ninety-eight woody plant species were registered, grouped in 84 genera and 35 families of angiosperms. The most diverse families were Fabaceae and Euphorbiaceae. The predominant life form were trees $(75.5 \%)$. This tropical forest shows a high richness and species turnover. The dominant species according to the RIV were Resinanthus aromaticus and Brosimum alicastrum, although the set of six dominant species varied greatly between the study sites.

Conclusions: The presence of some key species such as Brosimum alicastrum, Resinanthus aromaticus and Sapranthus microcarpus allowed to identify the characteristic features of the semideciduous forest and its ecotones with the medium evergreen forest and the deciduous forest.

Key words: conservation, primary vegetation, relative importance value, Resinanthus aromaticus, seasonally dry tropical forests, species turnover. 


\section{INTRODUCCIÓN}

La selva mediana subcaducifolia ( $\mathrm{SMsC}$ ) sensu Miranda y Hernández X. (1963), presente en el estado de Veracruz, es un tipo de vegetación que se desarrolla en pequeños fragmentos dentro del principal bioma de la región, que es la selva tropical estacionalmente seca (STES). La STES incluye una variedad de comunidades vegetales que presentan una fenología adaptada a condiciones climáticas con cambios estacionales caracterizados por la presencia de un periodo seco prolongado (Rzedowski, 1978; Trejo, 1999; Dirzo, et al., 2011). Las variaciones geológicas, topográficas, fisiográficas y edáficas de la zona central de Veracruz generan una amplia variedad de características microclimáticas, debido a lo cual se presentan al menos otros cinco tipos de comunidades vegetales: selva mediana subperennifolia (SMsP), selva baja caducifolia (SBC), palmar, encinar y vegetación riparia o de galería, además de vegetación secundaria y pastizales naturales (CastilloCampos, 1995). Los fragmentos de SMsC suelen ser reducidos debido a que esta selva se presenta en sectores de laderas, hondonadas y barrancas donde la exposición de luz y la presencia de corrientes de agua generan condiciones de mayor humedad que en las áreas cercanas (Casti1lo-Campos, 1995). Estos manchones se encuentran amenazados por la sobreexplotación y el cambio de uso de suelo con fines agrícolas y ganaderos (Castillo-Campos, 1995; Palacios-Wassenaar et al., 2014); sin embargo, no están contemplados en alguna figura de protección (Ellis et al., 2011).

La SMsC de la zona central de Veracruz se caracteriza por tener un estrato arbóreo cerrado de 12-20 m de alto, estrato medio arbóreo abierto de 6-11 m, estrato arbustivo de 1-5 m, estrato herbáceo escaso y suelo cubierto con materia orgánica (Castillo-Campos, 1995; Palacios-Wassenaar et al., 2014). Presenta especies endémicas y amenazadas, incluidas en la Lista Roja de la IUCN (IUCN, 2012), tales como Resinanthus aromaticus (Cast.-Campos \& Lorence) Borhidi, Aspidosperma megalocarpon Müll. Arg., Hyperbaena jalcomulcensis E. Pérez \& Cast.-Campos y Pistacia mexicana Kunth, además de especies enlistadas en la Norma Oficial Mexicana
NOM-059-SEMARNAT-2010 (SEMARNAT, 2010) con alguna categoría de protección, como Astronium graveolens Jacq. y Beaucarnea inermis (S. Watson) Rose (Casti1lo-Campos, 1995; Palacios-Wassenaar et al., 2014).

Dada la velocidad a la que está desapareciendo la vegetación tropical en México (Masera et al., 1997; Trejo y Dirzo, 2000; Portillo-Quintero y Sánchez-Azofeifa, 2010) y particularmente la del estado de Veracruz (Krömer et al., 2010), es muy importante realizar estudios que amplíen el conocimiento sobre sus características. Por otro lado, la $\mathrm{SMsC}$ se presenta en áreas pequeñas y fragmentadas, con elevado riesgo de pérdida por cambio de uso del suelo (Castillo-Campos, 1995; Palacios-Wassenaar et al., 2014), por lo que también es importante identificar áreas con elevado valor de conservación, que podrían ser sometidas a algún tipo de régimen de protección.

El presente estudio tiene como objetivo describir la estructura, riqueza y diversidad de angiospermas leñosas de la $\mathrm{SMsC}$ en el centro de Veracruz, con la finalidad de contribuir al conocimiento de sus características e identificar áreas de alto valor para la conservación.

\section{Materiales y Métodos}

\section{Área de estudio}

Para este estudio se seleccionaron los fragmentos de $\mathrm{SMsC}$ en mejor estado de conservación presentes en los municipios Jalcomulco y Tlaltetela, de acuerdo con estudios previos en la zona (Castillo-Campos, 1995; PalaciosWassenaar et al., 2014). Estos municipios se localizan en la zona central del estado de Veracruz, México, entre los $19^{\circ} 17^{\prime}$ y $19^{\circ} 21^{\prime}$ de latitud norte y $96^{\circ} 42^{\prime}$ y $96^{\circ} 46^{\prime}$ de longitud oeste (Fig. 1). La zona de estudio es ambientalmente muy heterogénea debido a una orografía caracterizada por la presencia de mesetas planas y onduladas, barrancas, valles, cerros y lomeríos, con altitudes que oscilan entre los 350 y 900 m (Castillo-Campos, 1995). El grupo de suelos que predomina es el luvisol órtico con litosol, litosol con regosol eútrico y luvisol órtico, caracterizados como aluviones poco evolucionados, localizados en las barrancas, acantilados y laderas con pendientes pronun- 

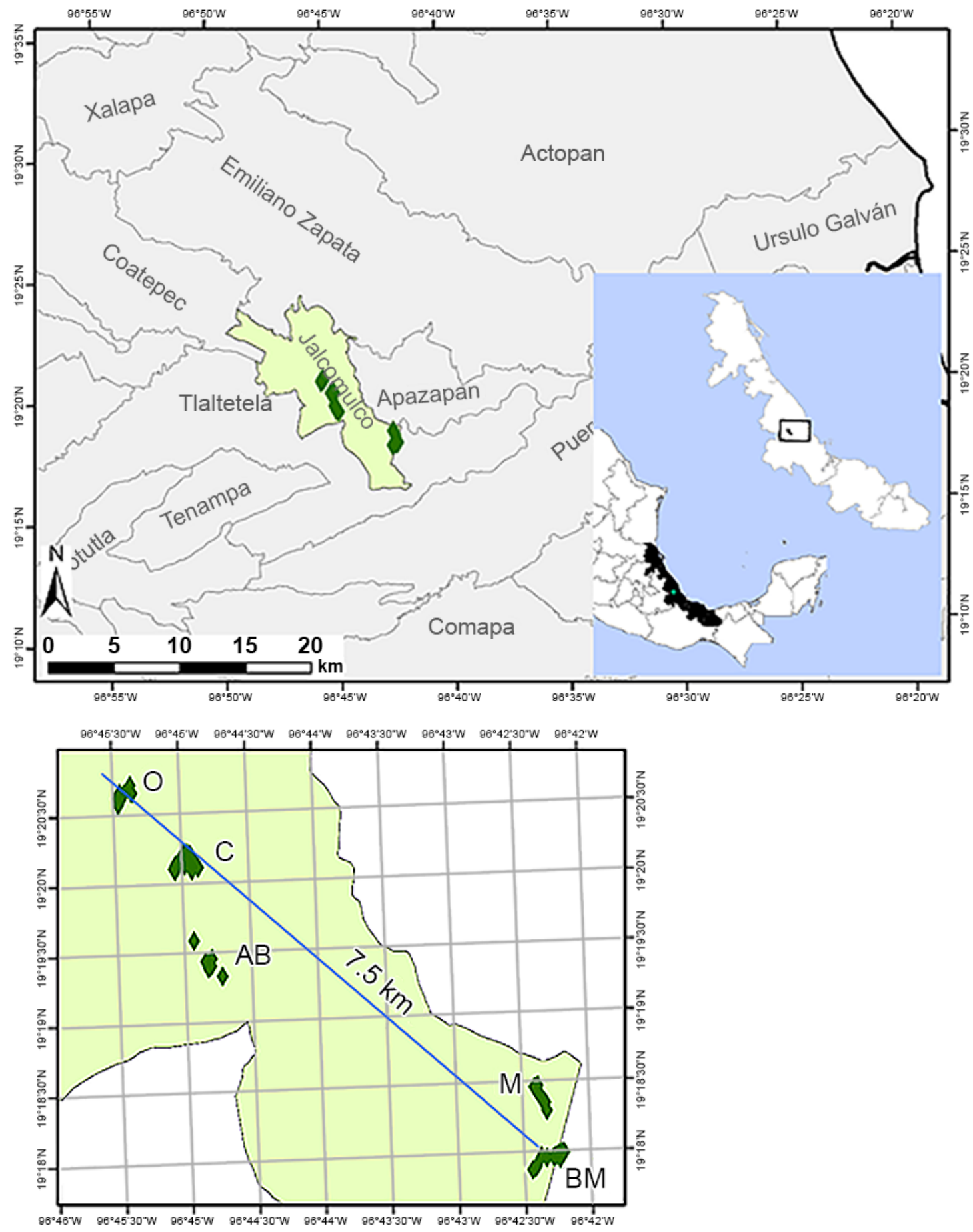

Figura 1: Localización geográfica de la zona de estudio y sitios de muestreo de la selva mediana subcaducifolia (SMsC) del centro de Veracruz en cinco sitios de estudio: Barranca de Monterrey (BM), El Manantial (M), Arroyo Blanco (AB), El Cerrito (C), El Ojital (O). 
ciadas (INEGI, 1984; Rossignol, 1987). En los macizos formados de roca caliza con intercalaciones de margas, se encuentran los litosoles calcáreos, los cuales están en proceso de carbonatación y descarbonatación; son suelos someros localizados en las grietas de las rocas y por lo tanto muy sensibles a la erosión (INEGI, 1984; Rossignol, 1987). En cuanto al clima, se presenta un gradiente entre semicálido húmedo a cálido subhúmedo, con una temperatura promedio anual de $24.6{ }^{\circ} \mathrm{C}$, precipitación promedio anual de $1088 \mathrm{~mm}$ y un periodo lluvioso concentrado en los meses de junio a septiembre. El patrón de lluvias manifiesta seis meses secos (precipitación $<60 \mathrm{~mm}$ ) y cuatro meses húmedos (precipitación $\geq 100 \mathrm{~mm}$ ) (SMN, 2012). Estas características corresponden a un clima cálido subhúmedo $\mathrm{Aw}_{1}$ con lluvias en verano (García, 2004) y a las condiciones que definen a la STES (Mooney et al., 1995; Trejo, 1999; Dirzo et al., 2011). Las áreas seleccionadas para el muestreo corresponden a cañadas, barrancas, laderas y hondonadas, donde las condiciones particulares de luz y humedad han permitido el desarrollo de la SMsC.

\section{Muestreo}

Durante abril de 2009 y hasta abril de 2010 se establecieron 67 parcelas de muestreo de las especies leñosas, de $100 \mathrm{~m}^{2}(10 \times 10 \mathrm{~m})$ cada una, a una distancia entre 25 y 50 $\mathrm{m}$ de acuerdo a la topografía y al tamaño de los fragmentos de la $\mathrm{SMsC}$, integrando una superficie total de 0.67 ha, distribuida en cinco sitios de estudio seleccionados por la presencia de la SMsC: Barranca de Monterrey (BM) con 15 parcelas, El Manantial (M) 12, Arroyo Blanco (AB) 20, El Cerrito (C) 10 y El Ojital (O) 10. El número de parcelas de cada sitio se definió de acuerdo con el tamaño del área de $\mathrm{SMsC}$, de tal manera que en los fragmentos con mayor superficie se estableció un mayor número de parcelas de muestreo, distribuidas sobre el área de manera representativa. En cada parcela se registró la altura y diámetro a la altura de pecho (DAP) (diámetro del tallo a $1.30 \mathrm{~m}$ de la base) de todos los individuos de plantas leñosas incluyendo lianas, enraizadas dentro de la parcela, con DAP igual o superior a $5 \mathrm{~cm}$. En el caso de las lianas, el DAP se midió a $1.3 \mathrm{~m}$ de la base del tallo. Se recolectaron muestras de las especies presentes y los ejemplares fueron herborizados y depositados para su identificación en el Herbario XAL del Instituto de Ecología, A.C. Para la actualización nomenclatural de los nombres científicos de la flora leñosa se utilizó la base de datos del Missouri Botanical Garden, a través de su página web (TROPICOS, 2017) y la clasificación del Angiosperm Phylogeny Group (APG IV, 2016).

\section{ANÁLISIS DE DATOS}

Se elaboró un inventario de las especies registradas en cada sitio de estudio y se estimó la riqueza total por sitio, el promedio por parcela (número de especies/0.01 ha), así como las características estructurales de la vegetación leñosa: densidad (individuos/ha), área basal promedio $\left(\mathrm{m}^{2} /\right.$ ha), altura promedio de todos los individuos (m) y altura máxima (m). Los valores se compararon mediante un análisis de varianza de una vía y se identificaron los grupos diferentes a través de un análisis comparativo de HolmSidak para los datos con distribución normal, mientras que los datos restantes se analizaron con el método de Kruskal-Wallis y comparaciones múltiples de Dunn. Estos análisis fueron realizados con apoyo del programa SigmaStat 3.5 (Systat Software, 2006).

La diversidad $\alpha$ de los sitios de estudio se estimó mediante el índice de $\alpha$ de Fisher, el cual se aproxima al número de especies con un individuo y presenta la ventaja de que no es afectado por el tamaño de la muestra cuando el número de individuos muestreados es superior a 1000. Esto permite establecer comparaciones de diversidad $\alpha$ entre estudios con diferentes áreas de muestreo (Magurran, 2004).

La importancia ecológica de las especies en cada sitio de estudio y en la SMsC en general se estimó mediante el Valor de Importancia Relativa de Curtis (VIR), el cual constituye un promedio entre la densidad relativa, el área basal relativa y la frecuencia relativa de cada especie registrada (Curtis y McIntosh, 1951; Mueller-Dombois y Ellenberg, 1974).

La diversidad $\beta$ de la SMsC en los cinco sitios de estudio se analizó en primer lugar mediante una matriz de datos de las especies por sitio y se estimaron dos indicadores: el coeficiente de Jaccard (Sneath y Sokal, 1973) 
con los datos de presencia-ausencia y el porcentaje de similitud entre sitios con los datos de abundancia. Ambos coeficientes se obtuvieron con el programa Multi Variate Statistical Package (MVSP) versión 3.1 (Kovach, 1999).

En segundo lugar, se integró una matriz de presencia-ausencia con el inventario de las especies en las 67 parcelas de la $\mathrm{SMsC}$ evaluada y se aplicó el coeficiente de Jaccard (Sneath y Sokal, 1973), donde el $0=100 \%$ de diferencia y el $1=100 \%$ de similitud entre las parcelas, utilizando el método de la media aritmética no ponderada (UPGMA), con el apoyo del mismo programa.

\section{Resultados}

\section{Estructura y diversidad alfa}

En las 67 parcelas muestreadas de los cinco sitios de estudio se registraron 1267 individuos con $\mathrm{DAP} \geq 5 \mathrm{~cm}$, perte- necientes a 98 especies de angiospermas leñosas, agrupadas en 83 géneros y 35 familias. Las familias con mayor riqueza de especies son: Fabaceae (10), Euphorbiaceae (9), Myrtaceae (8), Malpighiaceae y Moraceae (5) y Anacardiaceae, Apocynaceae, Capparaceae, Rubiaceae y Sapotaceae (4 cada una). Las primeras cinco familias incluyen $37.8 \%$ de las especies encontradas. Por otro lado, se detectaron 11 familias (31.4\%) representadas por una sola especie. La diversidad florística también se evidencia a nivel de género, ya que 75 de ellos (88.2\%) está representado por una sola especie. La relación especies/género es de 1.15. En cuanto a las formas de vida, predominan los árboles con $75.5 \%$ de las especies, mientras que los arbustos constituyen $15.3 \%$ y las lianas 9.2\%, demostrando así el buen estado de conservación de los fragmentos de vegetación de la $\mathrm{SMsC}$.

En el Cuadro 1 se presentan los valores de estructura y diversidad $\alpha$ de las especies en la $\mathrm{SMsC}$ en los cinco sitios de

Cuadro 1: Características del sitio, estructura y diversidad $\alpha$ de la selva mediana subcaducifolia (SMsC) del centro de Veracruz en cinco sitios de estudio: Barranca de Monterrey (BM), El Manantial (M), Arroyo Blanco (AB), El Cerrito (C), El Ojital (O). Valores con la misma letra no presentan diferencia significativa $(\mathrm{P}<0.05)$. $\mathrm{NO}=$ Noroeste, $\mathrm{S}=\mathrm{Sur}, \mathrm{SE}=$ Sureste. $\mathrm{DE}=$ Desviación Estándar.

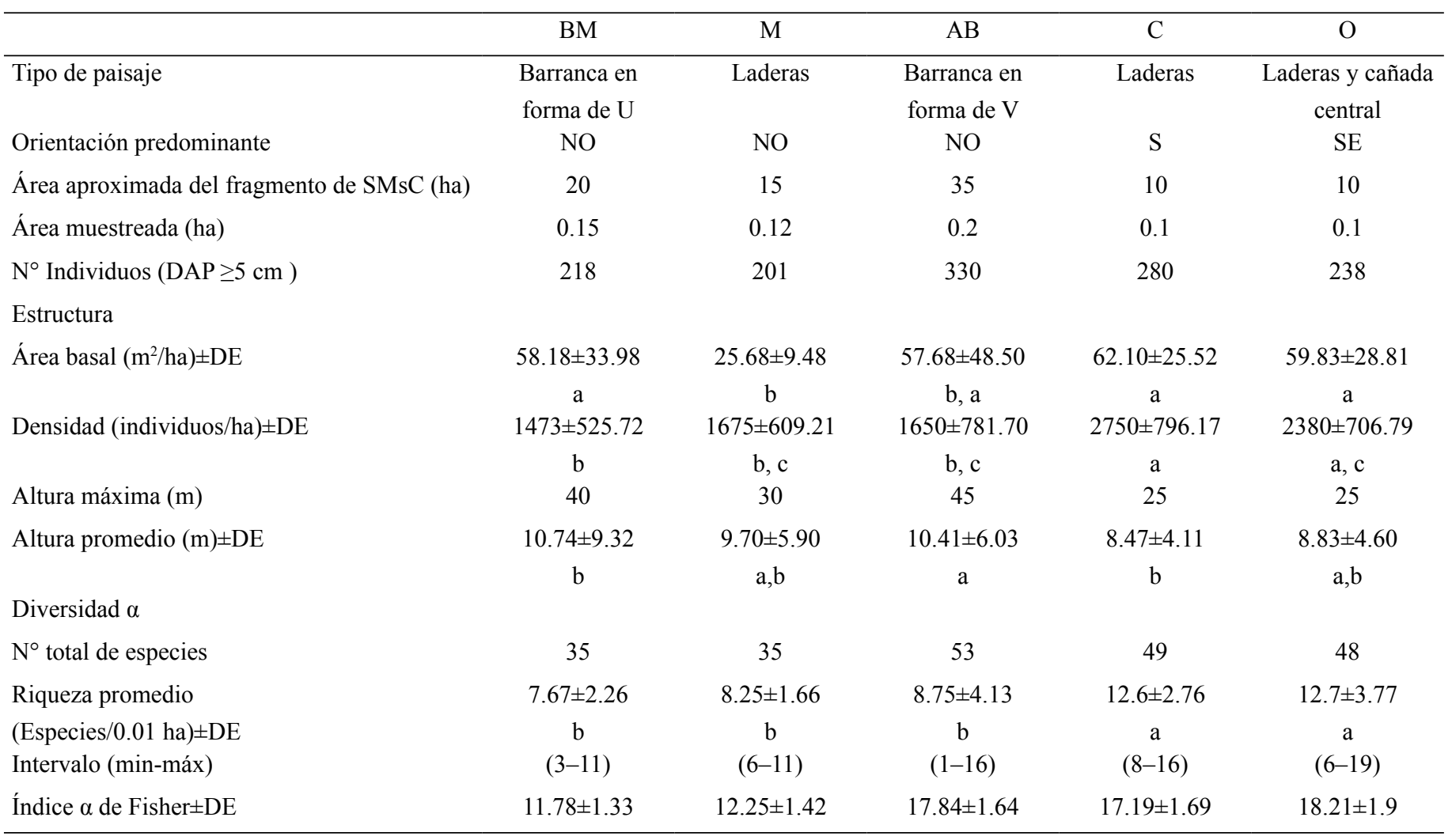


Cuadro 2: Valor de importancia de las seis especies principales de la selva mediana subcaducifolia (SMsC) del centro de Veracruz en cinco sitios de estudio: Barranca de Monterrey (BM), El Manantial (M), Arroyo Blanco (AB), El Cerrito (C), El Ojital (O). Entre paréntesis se presentan los valores relativos (\%).

\begin{tabular}{|c|c|c|c|c|}
\hline Sitio/Especie & $\begin{array}{c}\text { Densidad } \\
\text { Individuos/ha }\end{array}$ & $\begin{array}{c}\text { Área basal } \\
\mathrm{m}^{2} / \mathrm{ha}\end{array}$ & $\begin{array}{c}\text { Frecuencia } \\
\text { Número de parcelas de } \\
\text { estudio donde está presente }\end{array}$ & $\begin{array}{c}\text { Valor de Importancia } \\
\text { Relativa } \\
\text { (VIR) }\end{array}$ \\
\hline \multicolumn{5}{|l|}{ Barranca de Monterrey (BM) } \\
\hline Brosimum alicastrum $\mathrm{Sw}$. & $213.33(14.68)$ & $24.16(41.52)$ & $12(10.43)$ & 22.21 \\
\hline $\begin{array}{l}\text { Resinanthus aromaticus (Cast.-Campos \& Lorence) } \\
\text { Borhidi }\end{array}$ & $173.33(11.93)$ & $3.80(6.52)$ & $13(11.30)$ & 9.92 \\
\hline Sapranthus microcarpus (Donn. Sm.) R.E. Fr. & $186.67(12.84)$ & $1.62(2.79)$ & $10(8.70)$ & 8.11 \\
\hline Aphananthe monoica (Hemsl.) J.-F. Leroy & $100.00(6.88)$ & $5.45(9.37)$ & $9(7.83)$ & 8.02 \\
\hline Astronium graveolens Jacq. & $33.33(2.29)$ & $6.51(11.18)$ & $5(4.35)$ & 5.94 \\
\hline Comocladia engleriana Loes. & $86.67(5.96)$ & $0.70(1.20)$ & $6(5.22)$ & 4.13 \\
\hline Otras especies (29) & $660.00(45.41)$ & $15.96(27.43)$ & $60(52.17)$ & 41.67 \\
\hline Total BM & 1453.33 & 58.18 & 115 & 100.00 \\
\hline \multicolumn{5}{|l|}{ El Manantial (M) } \\
\hline $\begin{array}{l}\text { Resinanthus aromaticus (Cast.-Campos \& Lorence) } \\
\text { Borhidi }\end{array}$ & $375.00(22.39)$ & $6.46(25.17)$ & $12(12.12)$ & 19.89 \\
\hline Brosimum alicastrum $\mathrm{Sw}$. & $283.33(16.92)$ & $3.11(12.12)$ & $10(10.10)$ & 13.05 \\
\hline Aphananthe monoica (Hemsl.) J.-F. Leroy & $41.67(2.49)$ & $3.22(12.55)$ & $4(4.04)$ & 6.36 \\
\hline Mosannona depressa (Baill.) Chatrou & $125.00(7.26)$ & $0.77(2.99)$ & $6(6.06)$ & 5.50 \\
\hline Comocladia engleriana Loes. & $83.33(4.98)$ & $0.47(1.83)$ & $8(8.08)$ & 4.96 \\
\hline Ocotea tampicensis (Meisn.) Hemsl. & $50.00(2.99)$ & $1.14(4.43)$ & $6(6.06)$ & 4.49 \\
\hline Otras especies (29) & $716.67(42.79)$ & $10.50(40.90)$ & $53(53.54)$ & 45.74 \\
\hline Total M & 1675.00 & 25.68 & 99 & 100.00 \\
\hline \multicolumn{5}{|l|}{ Arroyo Blanco (AB) } \\
\hline $\begin{array}{l}\text { Resinanthus aromaticus (Cast.-Campos \& Lorence) } \\
\text { Borhidi }\end{array}$ & $315.00(19.09)$ & $21.35(37.24)$ & $20(11.43)$ & 22.59 \\
\hline Brosimum alicastrum $\mathrm{Sw}$. & $170.00(10.30)$ & $8.58(14.96)$ & $14(8.00)$ & 11.09 \\
\hline Sapranthus microcarpus (Donn. Sm.) R.E. Fr. & $110.00(6.67)$ & $1.06(1.84)$ & $12(6.86)$ & 5.12 \\
\hline Bursera simaruba (L.) Sarg. & $65.00(3.94)$ & $2.02(3.53)$ & $6(3.43)$ & 3.63 \\
\hline Licaria misantlae (Brandegee) Kosterm. & $50.00(3.03)$ & $2.62(4.56)$ & $4(2.29)$ & 3.29 \\
\hline Comocladia engleriana Loes. & $70.00(4.24)$ & $0.58(1.01)$ & $8(4.57)$ & 3.27 \\
\hline Otras especies (47) & $870.00(52.73)$ & $21.13(36.85)$ & $111(63.43)$ & 51.00 \\
\hline Total AB & 1650.00 & 57.34 & 175 & 100.00 \\
\hline \multicolumn{5}{|l|}{ El Cerrito (C) } \\
\hline $\begin{array}{l}\text { Resinanthus aromaticus (Cast.-Campos \& Lorence) } \\
\text { Borhidi }\end{array}$ & $260.00(9.42)$ & $20.84(33.55)$ & $9(7.38)$ & 16.78 \\
\hline Comocladia engleriana Loes. & $340.00(12.32)$ & $2.26(3.64)$ & $7(5.74)$ & 7.23 \\
\hline Yucca guatemalensis Baker & $200.00(7.25)$ & $1.51(2.44)$ & $8(6.56)$ & 5.41 \\
\hline
\end{tabular}


Cuadro 2: Continuación.

\begin{tabular}{lcccc}
\hline Sitio/Especie & $\begin{array}{c}\text { Densidad } \\
\text { Individuos/ha }\end{array}$ & $\begin{array}{c}\text { Área basal } \\
\mathrm{m}^{2} / \mathrm{ha}\end{array}$ & $\begin{array}{c}\text { Frecuencia } \\
\text { Número de parcelas de } \\
\text { estudio donde está presente }\end{array}$ & $\begin{array}{c}\text { Valor de Importancia } \\
\text { Relativa } \\
\text { (VIR) }\end{array}$ \\
\hline Adelia oaxacana (Müll. Arg.) Hemsl. & $200.00(7.25)$ & $0.76(1.22)$ & $7(5.74)$ & 4.74 \\
Bursera simaruba (L.) Sarg. & $160.00(5.80)$ & $2.01(3.24)$ & $5(4.10)$ & 4.38 \\
Mosannona depressa (Baill.) Chatrou & $140.00(5.07)$ & $1.67(2.68)$ & $6(4.92)$ & 4.22 \\
Otras especies (43) & $1460.00(52.90)$ & $33.05(53.22)$ & $80(65.57)$ & 57.23 \\
Total C & 2760.00 & 62.10 & 122 & 100.00 \\
\hline El Ojital (O) & & & & 14.64 \\
\hline Brosimum alicastrum Sw. & $480.00(20.17)$ & $10.45(17.46)$ & $8(6.30)$ & 10.94 \\
Resinanthus aromaticus (Cast.-Campos \& Lorence) & $200.00(8.40)$ & $9.91(16.56)$ & $10(7.87)$ & 5.11 \\
Borhidi & $100.00(4.20)$ & $4.30(7.19)$ & $5(3.94)$ & 4.40 \\
Licaria misantlae (Brandegee) Kosterm. & $50.00(2.10)$ & $5.71(9.53)$ & $2(1.57)$ & 4.35 \\
Discocnide mexicana (Liebm.) Chew & $60.00(2.52)$ & $3.95(6.60)$ & $5(3.94)$ & 3.95 \\
Bursera simaruba (L.) Sarg. & $60.00(2.52)$ & $3.23(5.40)$ & $5(3.94)$ & 56.59 \\
Exothea paniculata (Juss.) Radlk. & $1430.00(60.80)$ & $22.29(37.25)$ & $92(72.44)$ & 100.00 \\
Otras especies (42) & 2380.00 & 59.84 & 127 & \\
Total O & & & \\
\hline
\end{tabular}

estudio. En cuanto a atributos estructurales, $\mathrm{C}$ y O destacan por el área basal (Kruskal-Wallis $\mathrm{H}=17.168, \mathrm{P}=0.002$; comparación múltiple de Dunn) y densidad (ANOVA F=7.347, $\mathrm{P}<0.001$; comparación múltiple de Holm Sidak). AB destaca por la altura del dosel, tanto máxima como promedio (Kruskal-Wallis $\mathrm{H}=17.206, \mathrm{P}=0.002$; comparación múltiple de Dunn). La riqueza de las especies en la $\mathrm{SMsC}$ también presentó variaciones entre los sitios de estudio: $\mathrm{AB}$, con 53 especies en una superficie de 0.2 ha, fue el sitio con mayor riqueza total, seguido por $\mathrm{C}$ y $\mathrm{O}$, con 49 y 48 especies en una superficie de 0.1 ha respectivamente. La comparación estadística por parcela demuestra que $\mathrm{C}$ y $\mathrm{O}$ presentan una riqueza promedio (especies/0.01 ha) mayor que $\mathrm{BM}, \mathrm{M}$ y $\mathrm{AB}(\mathrm{F}=6.941 ; \mathrm{P}<0.001)$. El índice de $\alpha$ de Fisher confirma que la diversidad de especies es mayor en $\mathrm{C}, \mathrm{AB}$ y $\mathrm{O}$.

\section{Valor de importancia relativa (VIR)}

El listado de todas las especies registradas y su valor de importancia relativa (VIR) general y en cada sitio de estu- dio se presenta en el Apéndice. El conjunto de las seis especies con mayor VIR (Cuadro 2) es diferente en cada sitio de estudio. Las especies más importantes son Resinanthus aromaticus y Brosimum alicastrum Sw. que alcanzan los dos primeros lugares del VIR en todos los sitios a excepción de $\mathrm{C}$, donde $B$. alicastrum no se encuentra entre los primeros seis lugares. En $\mathrm{C}$ el segundo lugar de importancia es ocupado por Comocladia engleriana Loes., la cual se presenta entre las más importantes en todos los sitios, a excepción de O. Bursera simaruba (L.) Sarg. destaca entre las más importantes en $\mathrm{AB}, \mathrm{C}$ y $\mathrm{O}$, mientras que Aphananthe monoica (Hemsl.) J.-F. Leroy lo es en BM y M. Sapranthus microcarpus (Donn. Sm.) R.E. Fr. destaca en BM y AB y Mosannona depressa (Baill.) Chatrou en $\mathrm{M}$ y C. La importancia de las especies en cada sitio también varía en relación con sus atributos estructurales: Aphananthe monoica, Astronium graveolens, Brosimum alicastrum, Discocnide mexicana (Liebm.) Chew y Resinanthus aromaticus son importantes por su área basal, 
mientras que Sapranthus microcarpus y Mosannona depressa destacan por su densidad y Comocladia engleriana suele ser importante tanto por su densidad como por su frecuencia.

\section{Diversidad beta}

En el Cuadro 3 se presentan los índices de diversidad $\beta$ (Jaccard y porcentaje de similitud) para los cinco sitios de estudio. La mayoría de los valores encontrados son inferiores a 0.5 indicando que la $\mathrm{SMsC}$ en el área presenta una alta tasa de recambio de especies. De acuerdo con el índice de Jaccard estimado con los datos de presenciaausencia, los sitios más similares entre sí son $\mathrm{AB}$ y $\mathrm{O}$.

$\mathrm{El}$ dendrograma de todas las parcelas de la $\mathrm{SMsC}$ basado en los datos de presencia-ausencia de especies (Fig. 2) las agrupa en tres conjuntos principales, que denominaremos G1, G2 y G3. E1 grupo G3 se separa a un nivel de $12 \%$ de similitud, es decir con un recambio de especies de $88 \%$ respecto a los otros dos grupos de ve- getación; mientras que G1 y G2 se separan a un nivel de $20 \%$ de similitud, es decir, con un recambio de especies de $80 \%$. El G1 agrupa 42 parcelas (62.7\%), incluye parcelas

Cuadro 3: Diversidad $\beta$ de la selva mediana subcaducifolia (SMsC) del centro de Veracruz en cinco sitios de estudio: Barranca de Monterrey (BM), El Manantial (M), Arroyo Blanco (AB), El Cerrito (C), El Ojital (O). El \% de similitud se localiza a partir de la diagonal hacia el extremo superior derecho y el índice de Jaccard, de la diagonal hacia el extremo inferior izquierdo. En negritas se indican los valores más altos de similitud y subrayados, los valores menores.

\begin{tabular}{lccccc}
\hline & BM & M & AB & C & O \\
\hline BM & $*$ & 51.43 & 54.55 & 30.95 & 40.96 \\
$\mathrm{M}$ & 0.346 & $*$ & 56.82 & 47.62 & 53.01 \\
$\mathrm{AB}$ & 0.375 & 0.397 & $*$ & 52.94 & $\mathbf{6 3 . 3 7}$ \\
$\mathrm{C}$ & 0.183 & 0.313 & 0.36 & $*$ & 59.79 \\
$\mathrm{O}$ & 0.258 & 0.361 & $\mathbf{0 . 4 6 4}$ & 0.426 & $*$ \\
\hline
\end{tabular}

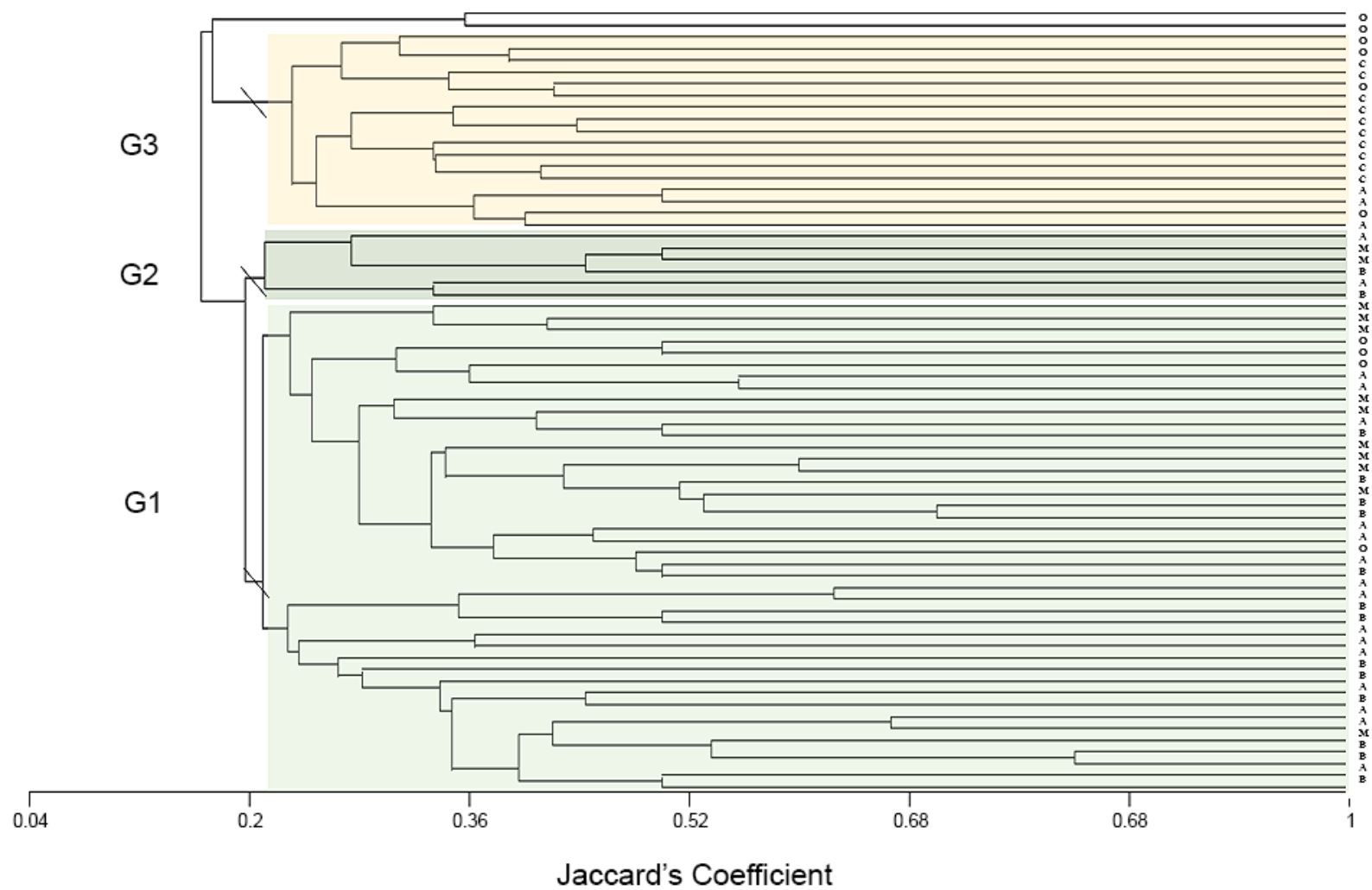

Figura 2: Dendrograma de similitud de la vegetación en las parcelas de la selva mediana subcaducifolia (SMsC) del centro de Veracruz. 
de cuatro sitios (BM, $\mathrm{M}, \mathrm{AB}$ y $\mathrm{O}$ ) e integra la mayor parte de las parcelas con semejanza florística igual o superior a $50 \%$. Está caracterizado por la presencia de Brosimum alicastrum, Resinanthus aromaticus, Aphananthe monoica y Sapranthus microcarpus en el estrato arbóreo, Acalypha villosa Jacq. en el estrato arbustivo y las lianas Pisonia aculeata L. y Mimosa guilandinae (DC.) Barneby. En este caso G1 es el grupo que mejor define a la $\mathrm{SMsC}$, por las especies dominantes que lo integran, destacando entre ellas la especie arbórea Resinanthus aromaticus que es endémica del centro del estado de Veracruz, incrementando con esto el valor de importancia para la conservación de estos fragmentos de vegetación. G2 agrupa seis parcelas $(8.9 \%)$ de sólo tres sitios (BM, M y AB), de las cuales sólo dos $(33 \%)$ presentan semejanza igual o superior a $50 \%$ y está caracterizado por la presencia de Manilkara zapota (L.) P. Royen, Resinanthus aromaticus, Brosimum alicastrum y Discocnide mexicana en el estrato arbóreo, Piper commutatum Steud. en el estrato arbustivo y no se presentan lianas. G3 incluye 17 parcelas $(23.9 \%)$ de tres sitios (AB, C y O), presenta sólo dos (11.8\%) parcelas con semejanza de $50 \%$ y las especies que lo caracterizan son Resinanthus aromaticus, Brosimum alicastrum, Comocladia engleriana y Yucca guatemalensis Baker en el estrato arbóreo, Euphorbia calcarata (Schltdl.) V.W. Steinm. en el estrato arbustivo; las lianas Hippocratea celastroides Kunth y Marsdenia coulteri Hemsl., y se presentan cactáceas columnares. Ninguno de los grupos de vegetación incluye parcelas de los cinco sitios. En la figura 3 se muestra el porcentaje de parcelas ubicadas en cada grupo de vegetación para los cinco sitios de estudio y se puede observar que $\mathrm{AB}$ es el único sitio que presenta parcelas en los tres grupos, mientras que $\mathrm{C}$ presenta la totalidad de sus parcelas en G3, destacándose como el sitio más seco.

La distribución del número de especies en los grupos G1, G2 y G3 se visualiza en el Diagrama de Venn (Fig. 4). Los tres grupos de vegetación comparten nueve especies: Resinanthus aromaticus, Brosimum alicastrum, Aphananthe monoica, Mosannona depressa, Sapranthus microcarpus, Bursera simaruba, Exothea paniculata (Juss.) Radlk., Discocnide mexicana y Piper commutatum. Los grupos G1 y G3 tienen adicionalmente 33 espe-

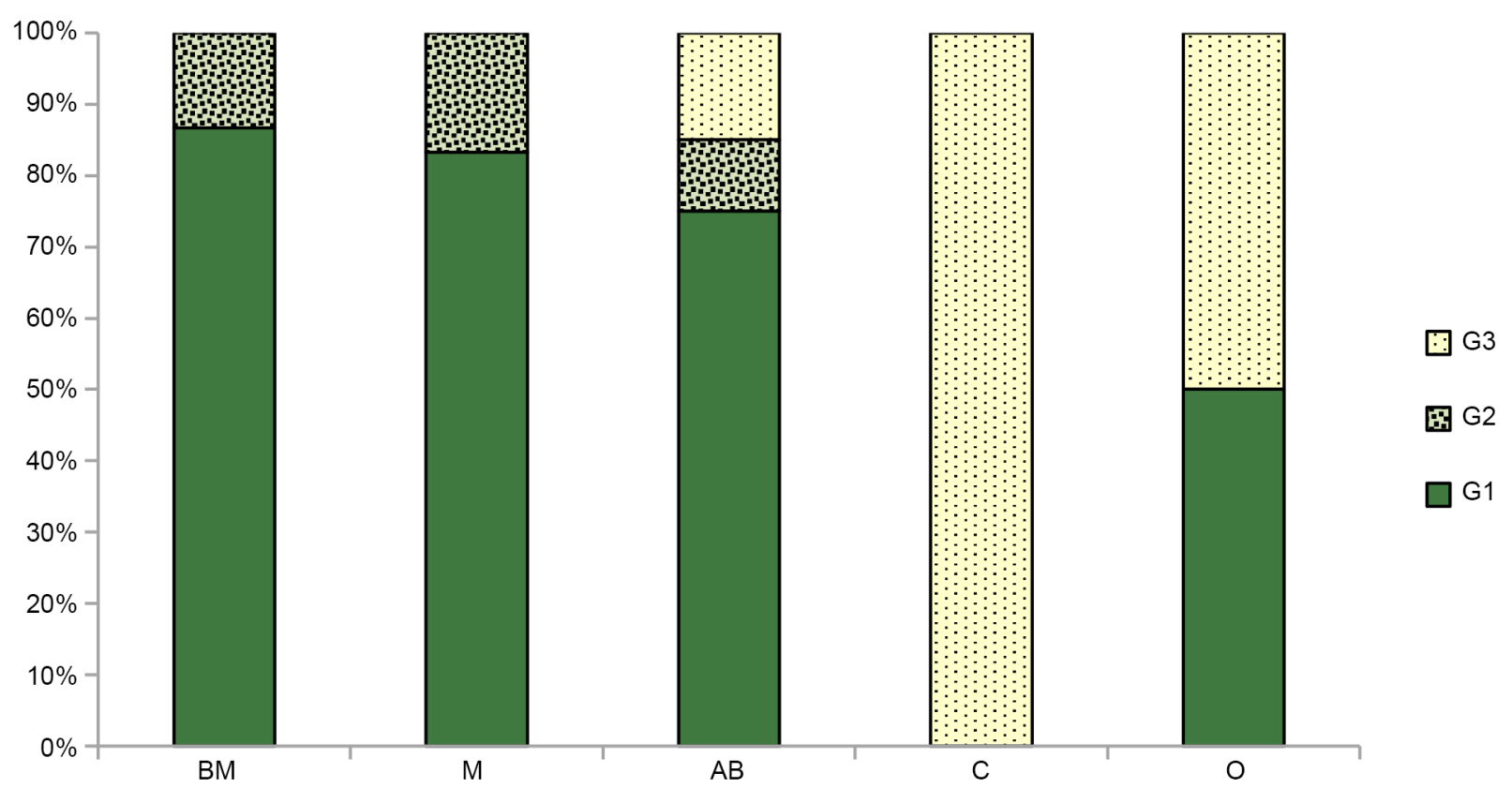

Figura 3: Porcentaje de parcelas en los grupos de similitud de la selva mediana subcaducifolia (SMsC) del centro de Veracruz en cinco sitios de estudio: Barranca de Monterrey (BM), El Manantial (M), Arroyo Blanco (AB), El Cerrito (C), El Ojital (O). 


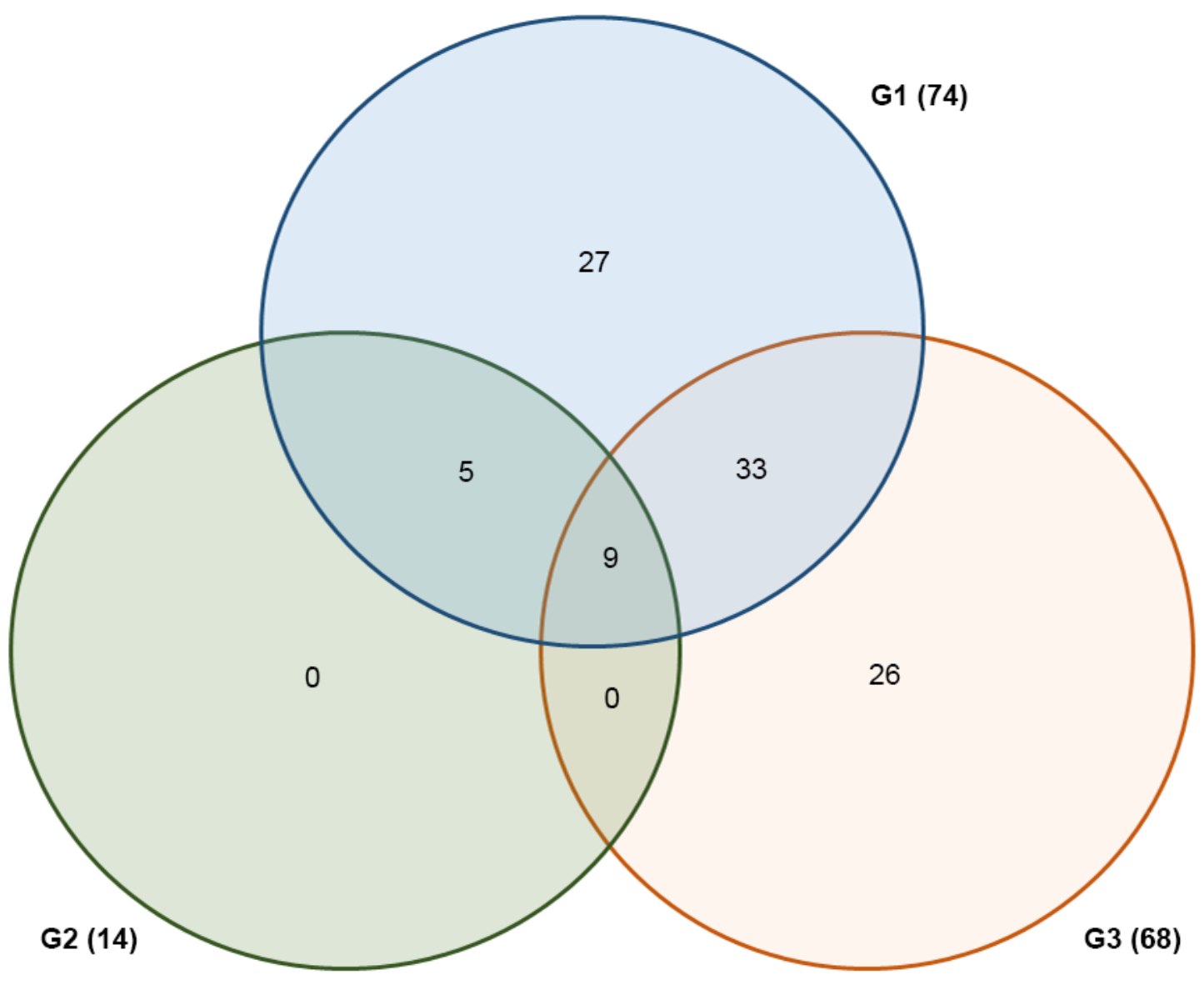

Figura 4: Diagrama de Venn de las especies de la selva mediana subcaducifolia (SMsC) del centro de Veracruz para los grupos de similitud. Entre paréntesis se indica el total de especies registradas en cada grupo.

cies en común, mientras que G1 y G2 comparten apenas cinco especies adicionales. Por otro lado, los grupos G2 y G3 no presentan especies adicionales en común. El grupo con mayor riqueza florística total es G1, con 74 especies, de las cuales 27 (36.5\%) son exclusivas de este grupo; seguido por el $\mathrm{G} 3$, con 68 especies, de las cuales 26 (38.2\%) son propias de este grupo. El grupo G2 tiene menor riqueza (14 especies) y carece de especies exclusivas.

En la figura 5 se presenta el porcentaje del VIR para algunas especies destacadas en los grupos G1, G2 y G3. El valor del VIR para las especies comunes a los tres grupos manifiesta diferencias importantes, que contribuyen a caracterizar a la $\mathrm{SMsC}$ de los mismos. En el caso de Resinanthus aromaticus, Brosimum alicastrum y Bursera simaruba, se observa que el VIR de estas es- pecies es similar en los tres grupos. En cambio, Aphananthe monoica, Mosannona depressa, Exothea paniculata, Discocnide mexicana y Piper commutatum, aunque son comunes a los tres grupos, se presentan con valores de VIR muy diferentes. Manilkara zapota, Discocnide mexicana y Piper commutatum son muy importantes en G2. Este grupo es el intermedio en el que dominan estas especies de follaje perenne que son características de la SMsP que se presenta solo en las zonas más húmedas del área de estudio (Castillo-Campos, 1995). Por otro lado, Comocladia engleriana está ausente en G2 y se presenta como dominante en G3, al igual que Yucca guatemalensis, Adelia oaxacana (Müll. Arg.) Hemsl. y Licaria misantlae (Brandegee) Kosterm., mientras que Ceiba aesculifolia (Kunth) Britten \& Baker f. es exclusiva de este 


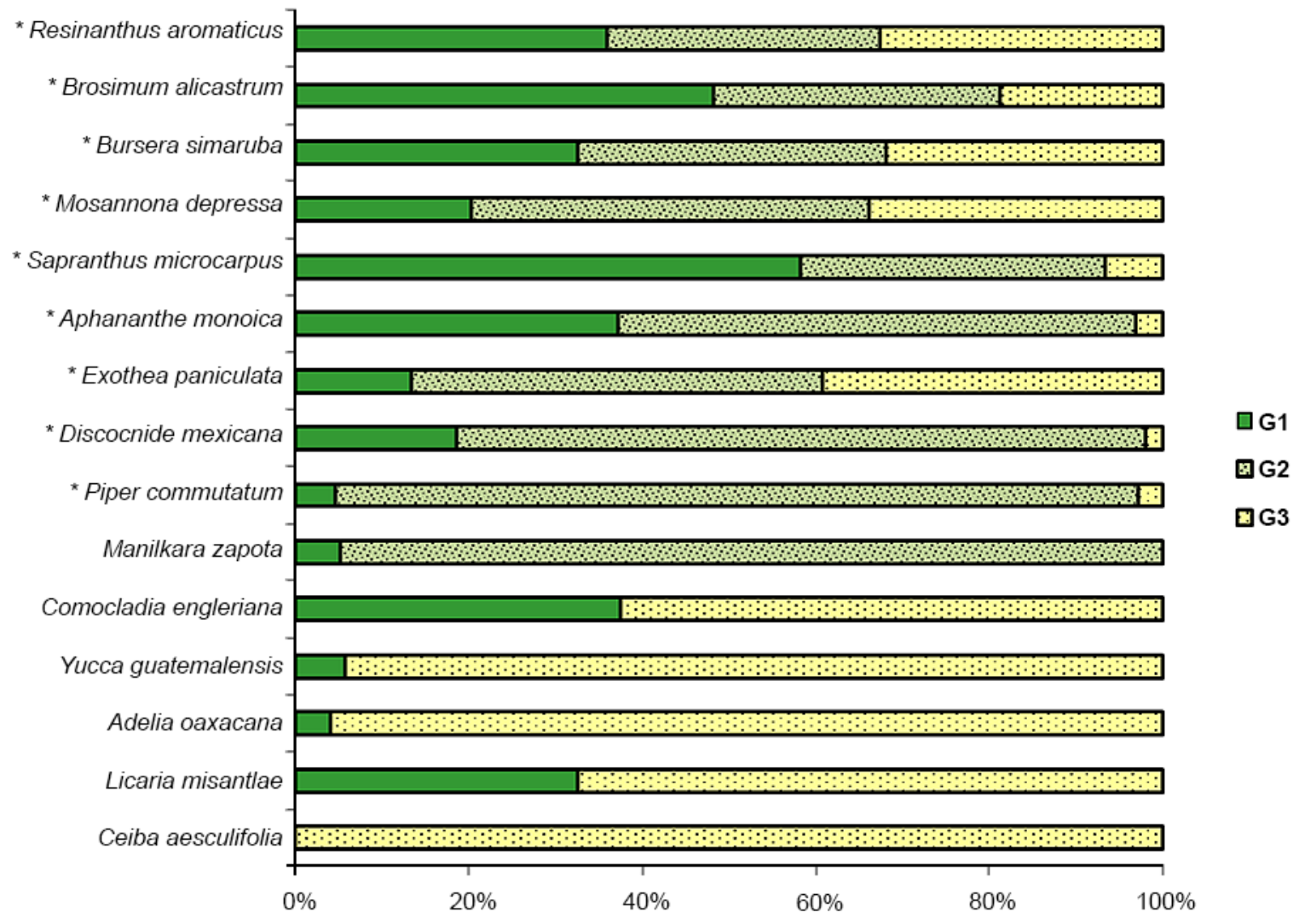

Figura 5: Comportamiento del Valor de Importancia Relativa de Curtis (VIR) de algunas especies destacadas en los grupos de similitud de la selva mediana subcaducifolia (SMsC) en el centro de Veracruz. Con asterisco se señalan las especies compartidas por los tres grupos.

grupo. Estas especies son comunes en la SBC del área de estudio (Castillo-Campos, 1995). En el grupo G1 se observa la dominancia de especies arbóreas que caracterizan a la $\mathrm{SMsC}$ de la zona de estudio, adicionales a $R e$ sinanthus aromaticus y Brosimum alicastrum, tales como Sapranthus microcarpus y Aphananthe monoica (Casti1lo-Campos, 1995; Palacios-Wassenaar et al., 2014).

\section{DIsCUSIÓN}

La riqueza total promedio de especies leñosas en los sitios de estudio de la SMsC (44) es similar o inferior a la registrada en áreas de conservación de la selva tropical estacionalmente seca (STES) en Costa Rica y Nicaragua, que fue de 44 a 75 especies (Gillespie et al., 2000; González-Rivas et al., 2006), aunque la diversidad de familias es mayor. Al comparar estos valores con otros estudios realizados en México, se observa que la riqueza de especies es inferior al promedio de 58.5 encontrado por Trejo y Dirzo (2002) para 20 sitios, y al total de 127 especies reportado por Bravo-Bolaños et al. (2016) para Nayarit; pero similar al promedio de 41.5 mencionado por Pineda-García et al., (2007) en Guerrero. En cambio, la riqueza de familias (35) es superior al promedio de 31.6 reportado por Trejo y Dirzo (2002) para México, al valor de 24 encontrado en Guerrero (Pineda-García et al., 2007) y de 32 en Hidalgo (Granados-Victorino et al., 2017). Sin embargo, tal como lo señalan Murphy y Lugo (1995), la diferencia entre las áreas muestreadas y el diámetro mínimo considerado en los diversos estudios (2.5, 5 y $10 \mathrm{~cm}$ de DAP), hace difícil establecer comparaciones directas, ya que si consideramos el número de especies registrado en el área total estudiada (98 especies con DAP $\geq 5 \mathrm{~cm}$ en $0.67 \mathrm{ha}$ ), la riqueza sería superior a la reportada por los autores mencionados y a la que encontró Gentry (1995) en su estudio sobre las 
selvas secas del Neotrópico ( 65 especies con DAP $\geq 2.5$ $\mathrm{cm}$ por ha). Por otro lado, la riqueza total encontrada es mayor a la reportada por Castillo-Campos (1995) para la zona (98 vs 54 especies). Tomando en cuenta que el referido trabajo consideró todas las formas de vida, incluyendo especies leñosas y herbáceas, podemos afirmar que la riqueza total en la presente investigación es mayor, probablemente debido a una mayor intensidad en el muestreo.

Otro indicador de la diversidad florística de esta $\mathrm{SMsC}$ es el elevado número de familias y géneros representados por pocas especies (dos o menos especies). La zona de estudio presenta $66.7 \%$ de familias con dos o menos especies, valor similar al registrado por Gillespie et al. (2000) para la STES de Costa Rica y Nicaragua y una relación especies/género de 1.15, comparable al valor de 1.17 registrado por Trejo y Dirzo (2002) para las STES de México. Estos valores confirman la alta diversidad florística que caracteriza a las STES (Gentry, 1995; Gillespie et al., 2000; Dirzo et al., 2011) y en este caso a la SMsC del centro de Veracruz. Cabe destacar que estudios recientes sobre las STES del Neotrópico destacan su riqueza y su especificidad de acuerdo con la región geográfica donde se ubican (DRYFLOR et al., 2016).

Las familias con mayor número de especies registradas son Fabaceae y Euphorbiaceae, lo cual coincide con diversos estudios en Centroamérica (p. ej. Gentry, 1995; Gillespie et al., 2000; González-Rivas et al., 2006) y en México (p. ej. Trejo y Dirzo, 2002; Pineda-García et al., 2007; Williams-Linera y Lorea, 2009; Bravo-Bolaños et al., 2016; Williams et al., 2017). También destaca la variabilidad del conjunto de especies dominantes en los sitios muestreados, coincidiendo con diversos trabajos sobre STES y sus tipos de vegetación asociados (p. ej. Gentry, 1995; Gillespie et al., 2000; Trejo y Dirzo, 2002; Dirzo et al., 2011; Dzib-Castillo et al., 2014; Bravo-Bolaños et al., 2016; DRYFLOR et al., 2016; Granados-Victorino et al., 2017).

Las especies pertenecientes a la $\mathrm{SMsC}$ en este estudio concuerdan con investigaciones previas realizadas en la zona (Robles, 1986; Castillo-Campos, 1995; Palacios-Wassenaar et al., 2014), que señalan a Brosimum alicastrum, Aphananthe monoica y Resinanthus aroma- ticus como especies dominantes en el estrato arbóreo, junto con Comocladia engleriana. Sin embargo, otras especies mencionadas en trabajos previos (Robles, 1986; Castillo-Campos, 1995) como características de la SMsC de la zona, por ejemplo Hyperbaena jalcomulcensis, no aparecen entre las dominantes en el presente estudio. Por otro lado, se presentan otras especies no mencionadas anteriormente como dominantes, tales como Yucca guatemalensis, Adelia oaxacana y Licaria misantlae. En cuanto al predominio de las especies, se observan importantes variaciones: el listado de las seis especies dominantes de acuerdo con su VIR fue diferente en todos los sitios, lo cual sugiere que la $\mathrm{SMsC}$ presenta una elevada tasa de recambio de especies en el área de estudio. Este hecho se confirma por los bajos valores de similitud encontrados entre parcelas, sitios muestreados y grupos de vegetación formados en el dendrograma, y coincide con lo reportado en investigaciones sobre STES en el Neotrópico (DRYFLOR et al., 2016), en México (Trejo y Dirzo, 2002) y sobre tipos de vegetación que se desarrollan dentro de la STES tales como el bosque tropical caducifolio y subcaducifolio (Bravo-Bolaños et al., 2016; Williams et al., 2017). También las especies compartidas presentan valores de importancia diferentes en cada sitio. Esta variabilidad en la composición florística y dominancia de las especies entre localidades ha sido destacada en numerosos trabajos como una característica distintiva de la STES en la cual se encuentra inmersa esta $\mathrm{SMsC}$, lo cual incrementa la necesidad de establecer mecanismos de protección para conservar los escasos remanentes de vegetación primaria de este tipo (p. ej. Gentry, 1995; Gillespie et al., 2000; Trejo y Dirzo, 2002; Dirzo et al., 2011; DRYFLOR et al., 2016).

La única especie encontrada en todos los muestreos en una investigación realizada en Costa Rica y Nicaragua es Bursera simaruba y ninguna especie fue dominante de manera consistente, lo cual parece apoyar la hipótesis de Gentry (1988), de que la dominancia de especies en las STES es impredecible y probablemente determinada por eventos estocásticos (Gillespie et al., 2000). Por otro lado, la marcada dominancia de Resinanthus aromaticus en el 
área de estudio podría corroborar lo señalado por Williams et al. (2010) en cuanto a que las especies endémicas de la STES se encuentran particularmente bien adaptadas a las condiciones locales y aportan una importante contribución a la estructura de la comunidad vegetal. Investigaciones recientes sobre la STES en el Neotrópico señalan que no hay especies dominantes comunes en áreas geográficamente separadas y que las especies características localmente abundantes y dominantes suelen tener una distribución restringida (DRYFLOR et al., 2016).

La presencia, abundancia y frecuencia y, en muchos casos, dominancia de acuerdo con el VIR de especies como Brosimum alicastrum, Bursera simaruba y Comocladia engleriana en estos tipos de vegetación ha sido reportada en diversos estudios en México (Rzedowski, 1978; Godínez-Ibarra y López-Mata, 2002; Pineda-García et al. 2007; Dzib-Castillo et al., 2014; Bravo-Bolaños et al., 2016; Williams et al., 2017).

Los estudios sobre la vegetación de la zona realizados por Castillo-Campos (1995) señalan la diferenciación de la vegetación en varios subtipos de acuerdo con la clasificación de Miranda y Hernández X. (1963): selva mediana subperennifolia ( $\mathrm{SMsP}),(\mathrm{SMsC})$ y selva baja caducifolia (SBC), de acuerdo con la proporción de especies caducifolias. La presencia y dominancia de ciertas especies características, adicionales a Brosimum alicastrum y Resinanthus aromaticus, permiten diferenciar estos tipos de vegetación. De esta manera, la dominancia de Aphananthe monoica, Sapranthus microcarpus y Astronium graveolens indican un predominio de la $\mathrm{SMsC}$ en $\mathrm{AB}, \mathrm{BM}$ y $\mathrm{M}$. Por otro lado, la presencia y dominancia de especies características de la SMsP, como Manilkara zapota, Protium copal (Schltdl. \& Cham.) Engl. y Piper commutatum indican una posible transición entre la $\mathrm{SMsC}$ y la SMsP en BM y AB. Esto puede explicarse debido a que $\mathrm{BM}$ y $\mathrm{AB}$ son sitios ubicados en cañadas profundas, donde hay áreas con menor exposición a la luz solar y con orientación predominante hacia el norte. En cambio, en $\mathrm{C}$ y $\mathrm{O}$ se observa la presencia y dominancia de especies como Bursera simaruba, Pistacia mexicana, Fraxinus dubia (Willd. ex Schult. \& Schult. f.) P.S.
Green \& M. Nee, Karwinskia humboldtiana (Schult.) Zucc., Beaucarnea inermis, Ceiba aesculifolia y cactáceas columnares, características de la SBC, que indican una transición entre la SMsC y este tipo de vegetación (Miranda y Hernández X., 1963; Castillo-Campos, 1995; Pineda-García et al., 2007). Las parcelas en estos sitios presentaron una orientación predominante hacia el sur y se encuentran sobre lomas y hondonadas poco profundas que permiten una mayor exposición a la luz solar, por lo que el grado de humedad es menor. La dominancia de Yucca guatemalensis, especie característica de la vegetación secundaria en la zona (Castillo-Campos, 1995), podría estar indicando un mayor grado de perturbación en $\mathrm{C}$, en comparación con los otros sitios de estudio.

Se puede afirmar que la vegetación de BM, M y $\mathrm{AB}$ es característica de la $\mathrm{SMsC}$ del área de estudio, con algunos elementos de la SMsP en los lugares más húmedos. Por contraste, las especies presentes en $\mathrm{C}$ y $\mathrm{O}$ son indicadoras de que en estos sitios se presenta una ecotonía mayor entre la $\mathrm{SMsC}$ y la $\mathrm{SBC}$. $\mathrm{AB}$ es el único sitio que presenta una $\mathrm{SMsC}$ bien definida y sus zonas de contacto (ecotonos) con la SMsP y SBC como se puede corroborar en la figura 3.

El valor del VIR para las especies comunes a los tres grupos de vegetación, que presentó diferencias interesantes, así como la distribución de especies compartidas y exclusivas entre los mismos, permiten caracterizar la vegetación de la zona de estudio. Especies como Resinanthus aromaticus, Brosimum alicastrum y Bursera simaruba, cuya dominancia en los tres grupos es similar, se definen como características de la $\mathrm{SMsC}$ en la zona de estudio. La presencia y dominancia de especies de zonas ligeramente más húmedas, como Manilkara zapota, Piper commutatum, Mosannona depressa y Discocnide mexica$n a$, indican una transición hacia la SMsP. Por otro lado, la presencia y dominancia de especies de áreas más secas como Comocladia engleriana, Yucca guatemalensis, Adelia oaxacana, Beaucarnea inermis y cactáceas columnares señalan la transición entre la SMsC y la SBC. Con base en los datos del presente estudio, se puede afirmar que la $\mathrm{SMsC}$ del centro de Veracruz está caracterizada 
por la presencia de las nueve especies compartidas: Resinanthus aromaticus, Brosimum alicastrum, Aphananthe monoica, Mosannona depressa, Sapranthus microcarpus, Bursera simaruba, Exothea paniculata, Discocnide mexicana y Piper commutatum.

Este trabajo constituye una contribución al conocimiento de la flora de la SMsC inmersa en ciertas áreas de la STES en el centro de Veracruz, que contiene especies arbóreas endémicas como Resinanthus aromaticus e Hyperbaena jalcomulcensis, entre otras, de gran valor científico y utilitario. Este conocimiento es clave para destacar la importancia de estos ecosistemas y sustentar oportunas medidas de manejo para la conservación de su biodiversidad, ya que la STES de México es reconocida por sus diferencias con otras STES neotropicales y destaca por su riqueza y abundancia de especies endémicas (DRYFLOR et al., 2016). Se sugiere la creación de áreas bajo régimen de protección especial que contengan al menos los fragmentos de $\mathrm{SMsC}$ en el área de estudio, principalmente $\mathrm{AB}$, con un plan de manejo que incluya la participación de los habitantes locales como garantes de su conservación.

\section{CONTRIBUCIÓN DE AUTORES}

OPW, GCC, SVT y MMA participaron en el muestreo de campo, OPW y GCC analizaron los datos, OPW redactó el texto, GCC, SVT y MMA revisaron el manuscrito. Todos los autores contribuyeron a la aprobación del manuscrito final.

\section{FINANCIAMIENTO}

Este estudio fue apoyado por el Consejo Nacional de Ciencia y Tecnología (CONACyT) (No. de becario 2239609) para la tesis doctoral del primer autor, y por el Instituto de Ecología, A.C. (GCC 2030-10134).

\section{AgRADECIMIENTOS}

Nuestro agradecimiento a las personas que apoyaron en el trabajo de campo: Luis Lagunes, César Carvajal, Samaria Armenta, Diego Kanchi y Melquiades Garrido. A Rosario Landgrave Ramírez por la elaboración del mapa. Al
Centro de Investigaciones Tropicales de la Universidad Veracruzana (CITRO) por el apoyo brindado. A Sergio Avendaño y Carlos Durán del herbario XAL, por su apoyo en la identificación de los especímenes botánicos. Se agradece igualmente a los revisores/as anónimos por sus valiosas observaciones y recomendaciones, que contribuyeron a enriquecer el manuscrito.

\section{LITERATURA CITADA}

APG IV. 2016. An update of the Angiosperm Phylogeny Group classification for the orders and families of flowering plants. Botanical Journal of the Linnean Society 181(1): 1-20. DOI: https://doi.org/10.1111/boj.12385

Bravo-Bolaños, O., A. Sánchez-González, J. de Nova-Vazquéz y N. Pavón-Hernández. 2016. Composición y estructura arbórea y arbustiva de la vegetación de la zona costera de Bahía de Banderas, Nayarit, México. Botanical Sciences 94(3): 603-623. DOI: https://dx.doi.org/10.17129/ botsci. 461

Castillo-Campos, G. 1995. Ecología del paisaje del municipio de Jalcomulco, Veracruz. Tesis de maestría. Facultad de Ciencias, Universidad Nacional Autónoma de México. México, D.F., México. 192 pp.

Curtis, J. y R. McIntosh. 1951. An upland forest continuum in the prairie forest border region of Wisconsin. Ecology 32(3): 476-496. DOI: https://doi.org/10.2307/1931725

Dirzo, R., H. Young, H. Mooney y G. Ceballos. 2011. Introduction. In: Dirzo, R., H. Young, H. Mooney y G. Ceballos (eds.). Seasonally dry tropical forest: Ecology and Conservation. Island Press. Washington, D.C., USA. Pp. XI-XII.

DRYFLOR, K. Banda-R., A. Delgado-Salinas, K. G. Dexter, R. Linares-Palomino, A. Oliveiro-Filho, D. Prado, M. Pullan, C. Quintana, R. Riina, G. M. Rodríguez M., J. Weintritt, P. Acevedo-Rodríguez, J. Adarve, E. Álvarez, A. Aranguren B., J. C. Arteaga, G. Aymard, A. Castaño, N. Ceballos-Mago, Á. Cogollo, H. Cuadros, F. Delgado, W. Devia, H. Dueñas, L. Fajardo, Á. Fernández, M. Á. Fernández, J. Franklin, E. H. Freid, L. A. Galetti, R. Gonto, R. González-M., R. Graveson, E. H. Helmer, Á. Idárraga, R. López, H. Marcano-Vega, O. G. Martínez, 
H. M. Maturo, M. McDonald, K. McLaren, O. Melo, F. Mijares, V. Mogni, D. Molina, N. P. Moreno, J. M. Nassar, D. M. Neves, L. J. Oakley, M. Oatham, A. R. Olvera-Luna, F. F. Pezzini, O. J. Reyes Domínguez, M. E. Ríos, O. Rivera, N. Rodríguez, A. Rojas, T. Särkinen, R. Sánchez, M. Smith, C. Vargas, B. Villanueva y R. Toby Pennington. 2016. Plant diversity patterns in neotropical dry forests and their conservation implications. Science 353(6306): 1383-1387. DOI: https://doi.org/10.1126/ science.aaf5080

Dzib-Castillo, B., C. Chanatásig-Vaca y N. A. GonzálezValdivia. 2014. Estructura y composición en dos comunidades arbóreas de la selva baja caducifolia y mediana subcaducifolia en Campeche, México. Revista Mexicana de Biodiversidad 85(1): 167-178. DOI: https:// dx.doi.org/10.7550/rmb.38706

Ellis, E., M. Martínez-Bello y R. Monroy-Ibarra. 2011. Focos rojos para la conservación de la biodiversidad. In: Comisión Nacional para el Conocimiento y Uso de la Biodiversidad (CONABIO) (ed.). La Biodiversidad en Veracruz: estudio de Estado Vol. I. Comisión Nacional para el Conocimiento y Uso de la Biodiversidad (CONABIO). México, D.F., México. Pp. 351-367.

García, E. 2004. Modificaciones al Sistema de Clasificación Climática de Köppen (para adaptarlo a las condiciones de la República Mexicana). 5a. ed. Instituto de Geografía, Universidad Nacional Autónoma de México. México, D.F., México. 90 pp.

Gentry, A. 1988. Changes in plant community diversity and floristic composition on environmental and geographical gradients. Annals of the Missouri Botanical Garden 75(1): 1-34. DOI: https://doi.org/10.2307/2399464

Gentry, A. 1995. Diversity and floristic composition of neotropical dry forests. In: Bullock, S., H. Mooney y E. Medina (eds.). Seasonally dry tropical forests. Cambridge University Press. Cambridge, UK. Pp. 146-194. DOI: https://doi.org/10.1017/CBO9780511753398.007

Gillespie, T., A. Grijalva y C. Farris. 2000. Diversity, composition, and structure of tropical dry forests in Central America. Plant Ecology 147(1): 37-47. DOI: https://doi.org/10.1023/a:1009848525399
Godínez-Ibarra, O. y L. López-Mata. 2002. Estructura, composición, riqueza y diversidad de árboles en tres muestras de selva mediana subperennifolia. Anales del Instituto de Biología, Universidad Nacional Autónoma de México, Serie Botánica 73(2): 283-314.

González-Rivas, B., M. Tigabu, K. Gerhardt, G. Castro-Marín y P. Odén. 2006. Species composition, diversity and local uses of tropical dry deciduous and gallery forests in Nicaragua. Biodiversity and Conservation 15(4): 1509 1527. DOI: https://doi.org/10.1007/s10531-005-2632-0

Granados-Victorino, R. L., A. Sánchez-González, D. MartínezCabrera y P. Octavio-Aguilar. 2017. Estructura y composición arbórea de tres estadios sucesionales de selva mediana subperennifolia del municipio de Huautla, Hidalgo, México. Revista Mexicana de Biodiversidad 88(1): 122135. DOI: https://doi.org/10.1016/j.rmb.2017.01.024

INEGI. 1984. Carta edafológica, escala 1: 250,000 Veracruz E 14-3. Instituto Nacional de Estadística, Geografía e Informática (INEGI). Aguascalientes, México.

IUCN. 2012. The IUCN Red List of Threatened Species, version 2012.2. http://www.iucnredlist.org (consultado abril de 2017).

Kovach, W. 1999. MVSP-a Multivariate Statistical Package for Windows, version 3.1. Kovach Computing Services. Pentraeth, UK.

Krömer, T., A. Acebey y A. Gómez-Pompa. 2010. Introducción. In: Gómez-Pompa, A., T. Krömer y R. Castro-Cortés (coord.). Atlas de la Flora de Veracruz, un patrimonio natural en peligro. Comisión del estado de Veracruz para la Conmemoración de la Independencia Nacional y la Revolución Mexicana, Gobierno del estado de Veracruz, Universidad Veracruzana. Xalapa, México. Pp. 25-42.

Magurran, A. 2004. Measuring biological diversity. Blackwell Science Ltd. Oxford, UK. 215 pp.

Masera, O., M. Ordóñez y R. Dirzo. 1997. Carbon emissions from Mexican forest: Current situation and long-term scenarios. Climatic Change 35(3): 265-295. DOI: https:// doi.org/10.1023/a:1005309908420

Miranda, F. y E. Hernández X. 1963. Los tipos de vegetación en México y su clasificación. Boletín de la Sociedad Botánica de México 28: 29-179. DOI: https://dx.doi.org/10.17129/ botsci. 1084 
Mooney, H., S. Bullock y E. Medina. 1995. Introduction. In: Bullock, S., H. Mooney y E. Medina (eds.). Seasonally dry tropical forests. Cambridge University Press. Cambridge, UK. Pp. 1-8. DOI: https://doi.org/10.1017/ CBO9780511753398.001

Mueller-Dombois, D. y H. Ellenberg. 1974. Aims and methods of vegetation ecology. John Wiley and Sons. New York, USA. 547 pp.

Murphy, P. y A. Lugo. 1995. Dry forest of Central America and the Caribbean. In: Bullock, S., H. Mooney y E. Medina (eds.). Seasonally dry tropical forest. Cambridge University Press. Cambridge, UK. Pp. 9-34. DOI: https:// doi.org/10.1017/CBO9780511753398.002

Palacios-Wassenaar, O., G. Castillo-Campos, S. M. VázquezTorres y S. del Amo-Rodríguez 2014. Flora vascular de la selva mediana subcaducifolia del centro de Veracruz, México. Revista Mexicana de Biodiversidad 85(1): 125142. DOI: https://doi.org//10.7550/rmb.34663

Pineda-García, F., L. Arredondo-Amezcua y G. IbarraManríquez. 2007. Riqueza y diversidad de especies leñosas del bosque tropical caducifolio El Tarimo, Cuenca del Balsas, Guerrero. Revista Mexicana de Biodiversidad 78(1): 129-139.

Portillo-Quintero, C. A. y G. A. Sánchez-Azofeifa. 2010. Extent and conservation of tropical dry forests in the Americas. Biological Conservation 143(1): 144-155. DOI: https:// doi.org/10.1016/j.biocon.2009.09.020

Robles, H. 1986. La vegetación y uso tradicional de las plantas de la barranca de Monte Rey, Municipio de Axocuapan, Ver., y sus alrededores. Tesis de licenciatura. Facultad de Biología, Universidad Veracruzana. Xalapa, Veracruz, México. 70 pp.

Rossignol, J. P. 1987. Morfoedafología del área Xalapa-Coatepec: Descripción de las unidades del mapa morfoedafológico Escala 1: 75,000. Office de la Recherche Scientifique et Technique Outre-Mer (ORSTOM)-Instituto Nacional de Investigación sobre Recursos Bióticos (INIREB). Xalapa, México.

Rzedowski, J. 1978. La Vegetación de México. Limusa. México, D.F., México. 432 pp.

SEMARNAT. 2010. Norma Oficial Mexicana NOM-059SEMARNAT-2010. Protección ambiental-Especies nativas de México de flora y fauna silvestres-Categorías de riesgo y especificaciones para su inclusión, exclusión o cambio-Lista de especies en riesgo. Secretaría del Medio Ambiente y Recursos Naturales. Diario Oficial de la Federación. Cd. Mx., México. http://dof.gob.mx/nota detalle.php? codigo $=5173091 \&$ fecha $=30 / 12 / 2010$

SMN. 2012. Servicio Meteorológico Nacional. http://www. smn.cna.gob.mx/SMN.html (consultado abril de 2012).

Sneath, P. H. A. y R. R. Sokal. 1973. Numerical taxonomy: the principles and practice of numerical classification. W. H. Freeman and Company. San Francisco, USA. 573 pp.

Systat Software. 2006. Systat Software, Inc. Sigmastat 3.5 for Windows. Tulsa, USA.

Trejo, I. 1999. El clima de la selva baja caducifolia en México. Investigaciones Geográficas 1(39): 40-52. DOI: https:// dx.doi.org/10.14350/rig.59082

Trejo, I. y R. Dirzo. 2000. Deforestation of seasonally dry tropical forest: a national and local analysis in Mexico. Biological Conservation 94(2): 133-142. DOI: https://doi. org/10.1016/s0006-3207(99)00188-3

Trejo, I. y R. Dirzo. 2002. Floristic diversity of Mexican seasonally dry tropical forests. Biodiversity and Conservation 11(11): 2063-2084. DOI: https://doi. org/10.1023/a:1020876316013

TROPICOS. 2017. Tropicos.org. Missouri Botanical Garden. http://www.tropicos.org/Name/29400090 ～(consultado mayo de 2017).

Williams, J. N., I. Trejo y M. W. Schwartz. 2017. Commonness, rarity, and oligarchies of woody plants in the tropical dry forests of Mexico. Biotropica 49(4): 493-501. DOI: https://doi.org/10.1111/btp.12447

Williams, J., J. Viers y M. Schwartz. 2010. Tropical dry forest trees and the relationship between local abundance and geographic range. Journal of Biogeography 37(5): 951-959. DOI: https://doi.org/10.1111/j.13652699.2009.02248.x

Williams-Linera, G. y F. Lorea. 2009. Tree species diversity driven by environmental and anthropogenic factors in tropical dry forest fragments of central Veracruz, Mexico. Biodiversity and Conservation 18(12): 3269-3293. DOI: https://doi.org/10.1007/s10531-009-9641-3 
Apéndice: Densidad, área basal, frecuencia y valor de importancia relativa (VIR) de las especies en la selva mediana subcaducifolia (SMsC) del centro de Veracruz. Las especies están ordenadas de acuerdo con el VIR general para el área de estudio. Se indica igualmente el VIR obtenido por las especies en los cinco sitios de estudio: Barranca de Monterrey (BM), El Manantial (M), Arroyo Blanco (AB), El Cerrito (C), El Ojital (O). En negritas y con asterisco se destacan las especies presentes en los cinco sitios de estudio. Colector: OMPW=Olivia M. Palacios-Wassenaar.

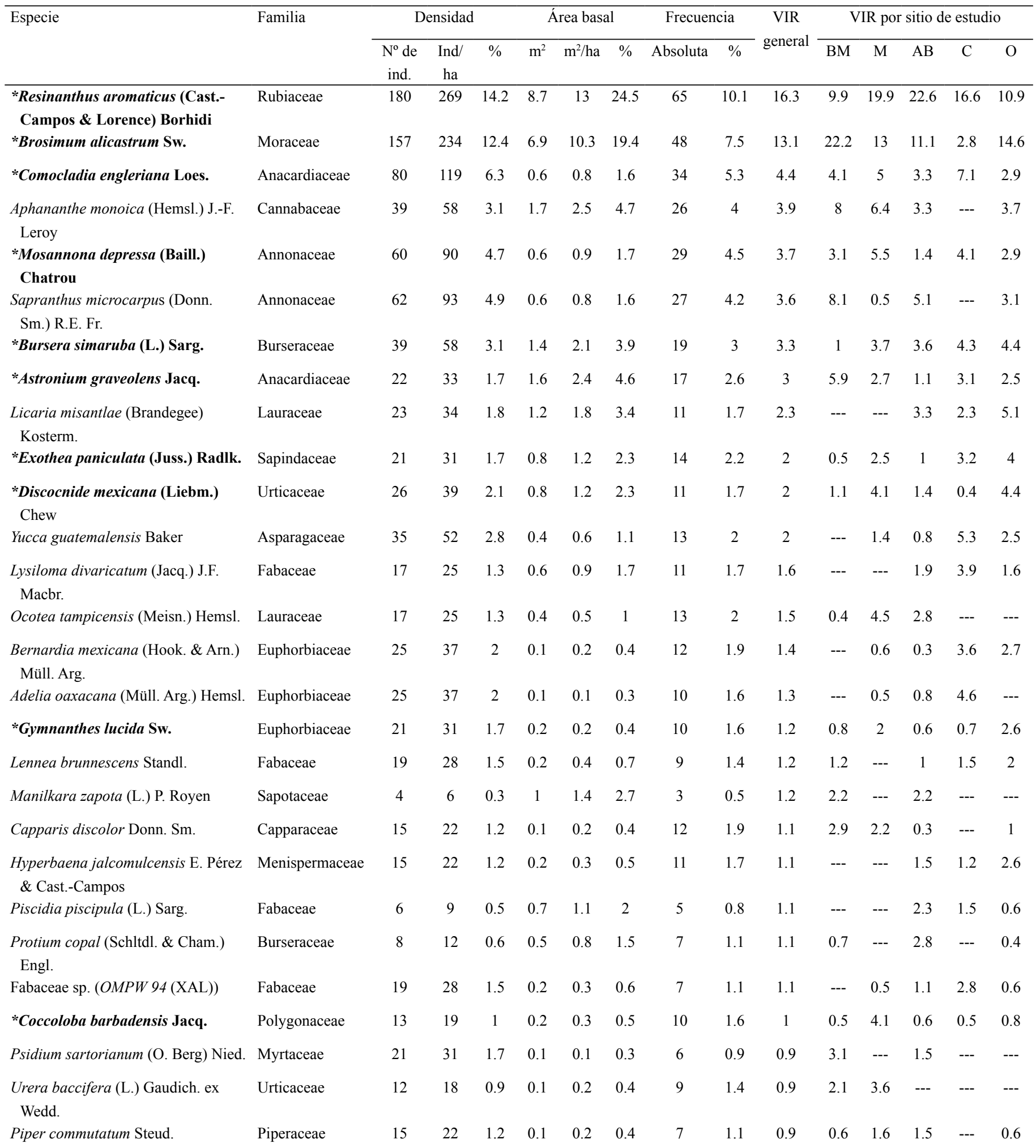


Apéndice: Continuación.

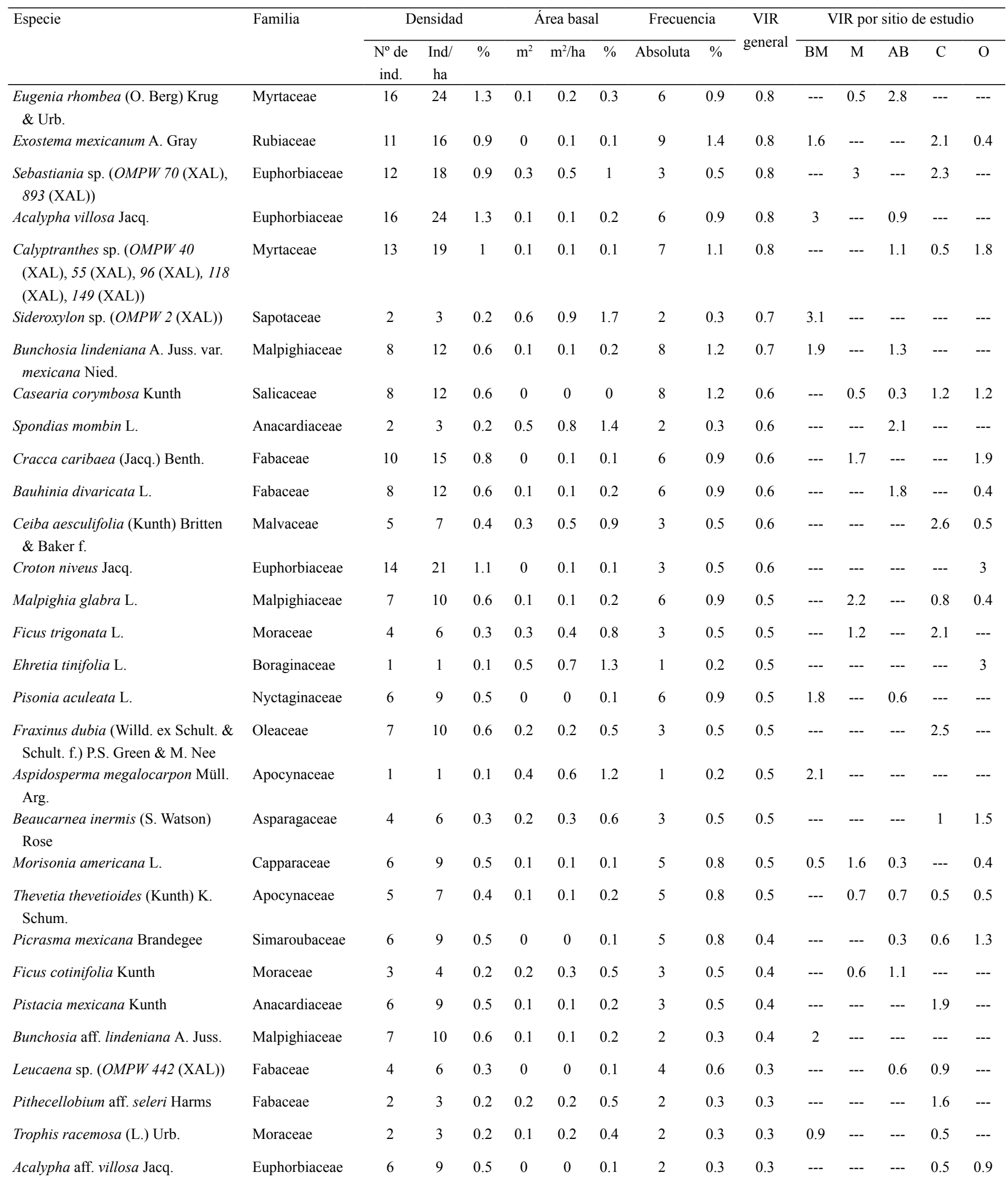


Apéndice: Continuación.

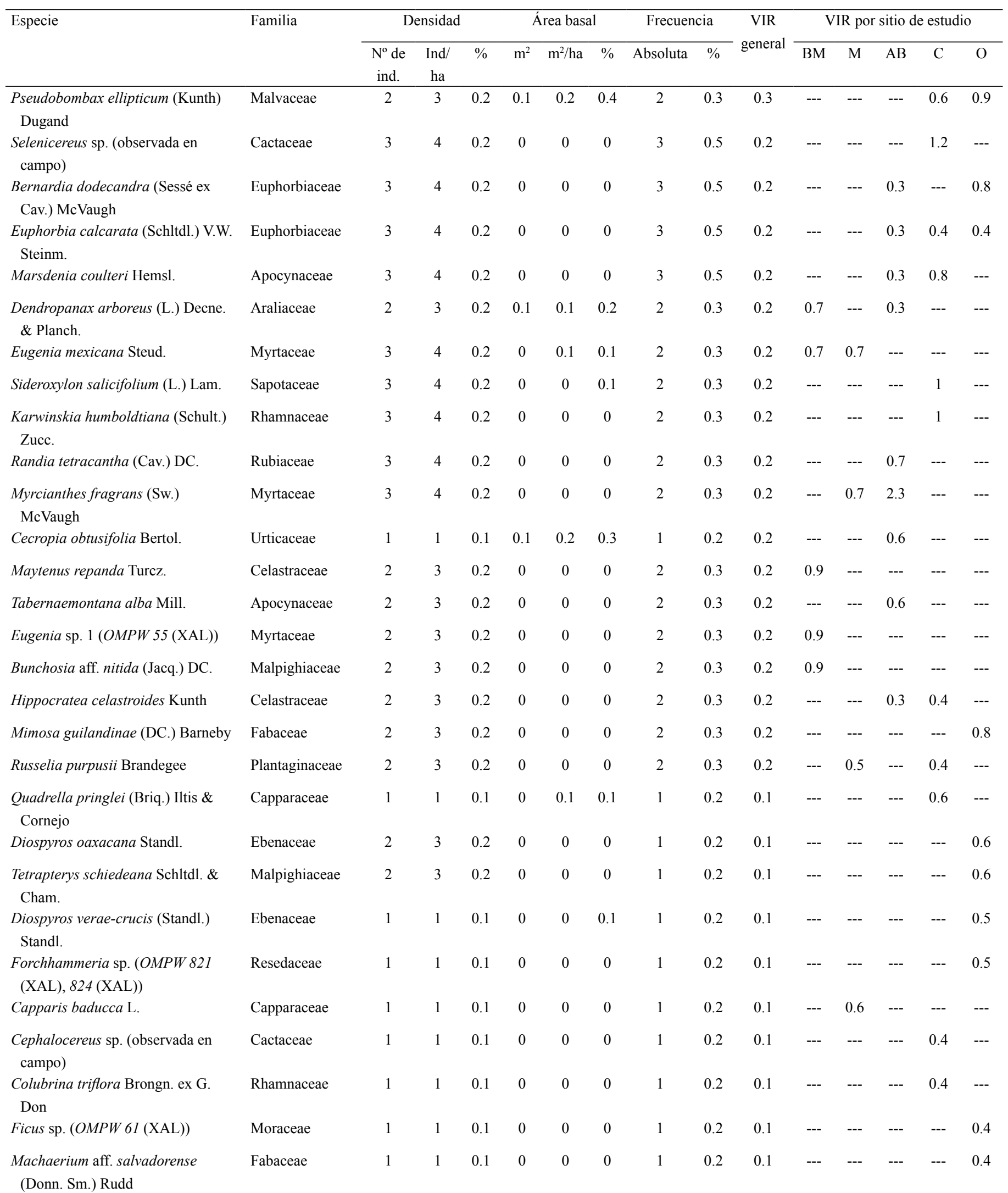


Apéndice: Continuación.

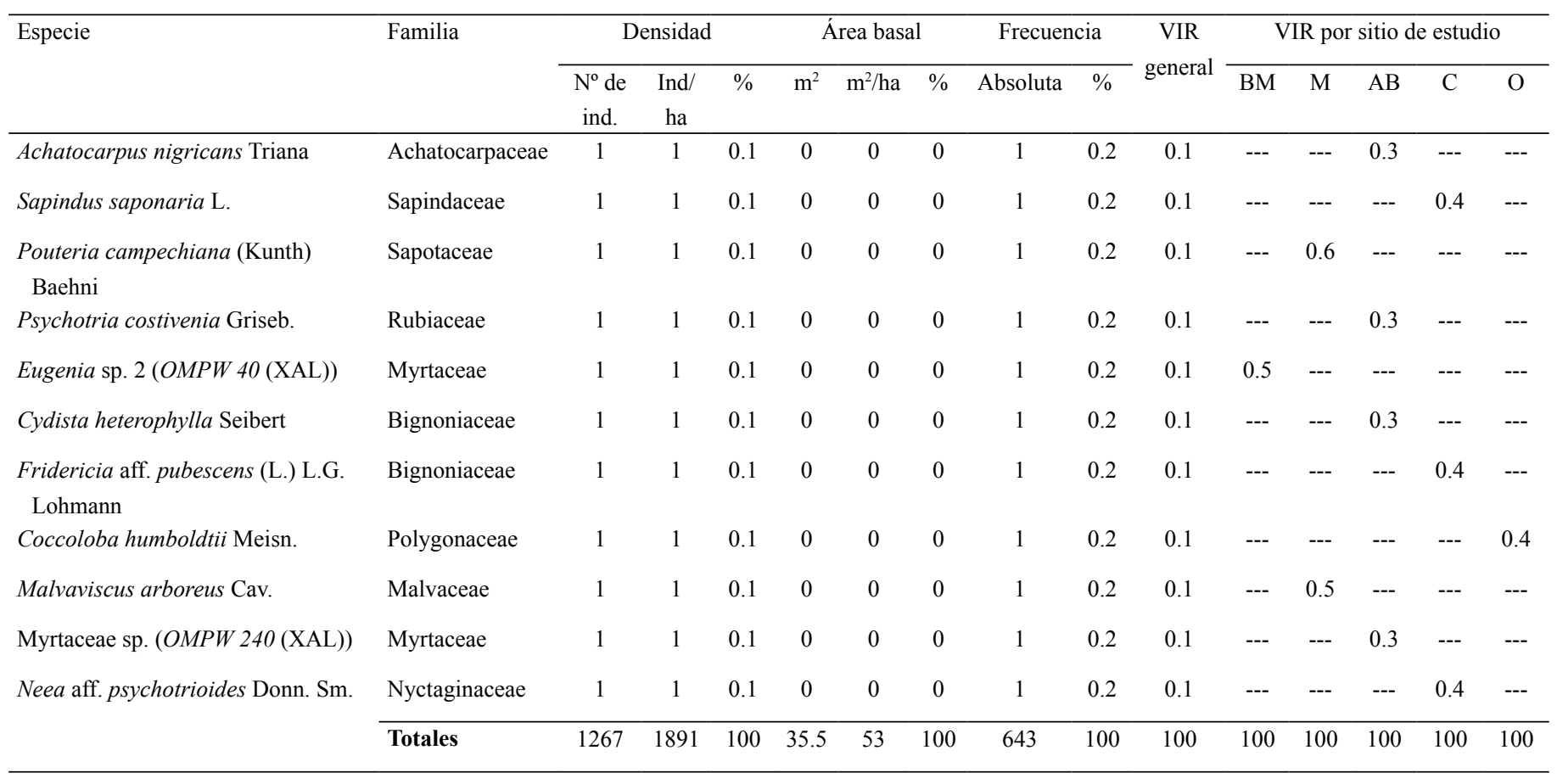

